\title{
The Hippo pathway regulates stem cell proliferation, self-renewal, and differentiation
}

\author{
Huan Liu, Dandan Jiang, Fangtao Chi, Bin Zhao ${ }^{\bowtie}$ \\ Life Sciences Institute, Zhejiang University, Hangzhou 310058, China \\ $\triangle$ Correspondence: binzhao@zju.edu.cn \\ Received March 12, 2012 Accepted March 15, 2012
}

\begin{abstract}
Stem cells and progenitor cells are the cells of origin for multi-cellular organisms and organs. They play key roles during development and their dysregulation gives rise to human diseases such as cancer. The recent development of induced pluripotent stem cell (iPSC) technology which converts somatic cells to stem-like cells holds great promise for regenerative medicine. Nevertheless, the understanding of proliferation, differentiation, and self-renewal of stem cells and organ-specific progenitor cells is far from clear. Recently, the Hippo pathway was demonstrated to play important roles in these processes. The Hippo pathway is a newly established signaling pathway with critical functions in limiting organ size and suppressing tumorigenesis. This pathway was first found to inhibit cell proliferation and promote apoptosis, therefore regulating cell number and organ size in both Drosophila and mammals. However, in several organs, disturbance of the pathway leads to specific expansion of the progenitor cell compartment and manipulation of the pathway in embryonic stem cells strongly affects their self-renewal and differentiation. In this review, we summarize current observations on roles of the Hippo pathway in different types of stem cells and discuss how these findings changed our view on the Hippo pathway in organ development and tumorigenesis.
\end{abstract}

KEYWORDS Hippo pathway, stem cell, YAP, Lats, differentiation, self-renewal

\section{INTRODUCTION}

Stem cells have the capacity to proliferate and differentiate into multiple cellular lineages, also defined as pluripotency. There are different classifications of stem cells that reflect the range of possible cell types they can produce and the ways from which the stem cells are derived. These include embryonic stem cells (ESCs), progenitor cells, mesenchymal stem cells (MSCs), and induced pluripotent stem cells (iPSCs). ESCs are derived from inner cell mass of blastocysts and can undergo extensive self-renewal in vitro and have the ability to undergo differentiation into all cell lineages, also called totipotency. Therefore ESCs are the cells of origin for multi-cellular organisms. Progenitor cells, which are derived from more developed fetal or adult tissues, are multipotent, meaning they give rise to more restricted lineages than ESCs. These potential lineages are usually determined by the tissue of origin. For example, liver progenitor cells or liver stem cells are capable of differentiating into cell types within a liver. MSCs are multipotent stem cells derived from tissue of mesoderm origin that can differentiate into a variety of cell types, including but not limited to: osteoblasts, chondrocytes, and adipocytes. However, MSCs do not have the capacity to reconstitute an entire organ. iPSCs are a type of pluripotent stem cells artificially derived from non-pluripotent cells, typically an adult somatic cell, for example by expression of a defined combination of transcription factors (Takahashi and Yamanaka, 2006). Due to the bypass of blastocyst as starting material, iPSCs are promising sources of stem cells for future medical use. More recently, studies of neoplastic tissues have provided evidence of self-renewing, stem-like cells within tumors, which have been called cancer stem cells (CSCs) (Reya et al., 2001). Despite rapid progress in generating stem-like cells from different sources and efforts in translating these cells to practical medical use, the underlying molecular mechanism of stem cell self-renewal, differentiation, and proliferation is not clear. Recently, the newly established Hippo signaling pathway was found to play important roles in each of these different types of stem cells. 
These findings will be summarized and discussed below.

\section{THE DROSOPHILA HIPPO PATHWAY}

Defined organ size is one of the most visible features of multi-cellular organisms. The regulation of organ size is a highly coordinated process governed by both physiological cues and an intrinsic mechanism. The underlying mechanism of organ autonomous size determination has remained largely unknown until the past decade. Extensive research has led to the identification of the Hippo tumor suppressor pathway as a key regulator of organ size in Drosophila and mammals (Pan, 2010). By genetic mosaic screens, mutation of the first batch of Hippo pathway genes warts (wts) (Justice et al., 1995; Xu et al., 1995), hippo (hpo) (Harvey et al., 2003; Jia et al., 2003; Pantalacci et al., 2003; Udan et al., 2003; Wu et al., 2003), and salvador (sav) (Kango-Singh et al., 2002; Tapon et al., 2002) were found to dramatically promote tissue overgrowth and organ size enlargement. These genes belong to the hyperplastic group of Drosophila tumor suppressors wherein mutations of these genes result in robust tissue overgrowth without alterations of cell fate determination or cell polarity (Hariharan and Bilder, 2006). Biochemical studies further revealed that Hpo directly interacts with Sav to phosphorylate and activate the complex formed by Wts and another core Hippo pathway protein, Mats (Wu et al., 2003; Lai et al., 2005; Wei et al., 2007) (Fig. 1A). The kinase activity of $\mathrm{Hpo}$ is antagonized by a newly identified PP2A phosphatase complex, dSTRIPAK (Ribeiro et al., 2010). By yeast two-hybrid, the Pan group identified the transcription co-activator Yorkie (Yki) as a Wts-interacting protein and a potent effector of the Hippo pathway (Huang et al., 2005). Subsequent biochemical studies showed that Wts directly phosphorylates and inhibits Yki (Dong et al., 2007). Scalloped $(\mathrm{Sd})$, the homolog of mammalian TEAD family transcription factors, were found to be critical partners of Yki in the regulation of gene expression (Goulev et al., 2008; Wu et al., 2008; Zhang et al., 2008; Zhao et al., 2008; Zhang et al., 2009a). Several transcriptional targets of the Hippo pathway have been identified, including cyclin $E$, which directly promotes cell cycle progression and cell proliferation, and diap1, which inhibits apoptosis (Tapon et al., 2002; Jia et al., 2003; Udan et al., 2003; Wu et al., 2003; Lai et al., 2005). The Hippo pathway also inhibits expression of a microRNA bantam to affect organ size, although the downstream effector of bantam is not clear. Furthermore, Yki-Sd was shown to transcriptionally induce $d M y c$, a potent promoter of ribosome biogenesis and cell growth (Neto-Silva et al., 2010; Ziosi et al., 2010), which may mediate the cell competition phenomenon observed in tissues with imbalance of Hippo pathway activity. In addition, Yki induces E2F1 (Goulev et al., 2008), which may be involved in cell-autonomous regulation of cell proliferation; the EGFR ligands Vein, Keren, and Spitz (Zhang et al., 2009b; Ren et al., 2010); the Jak/Stat pathway ligands
Unpaired1/2/3 (Upd1/2/3) (Karpowicz et al., 2010; Ren et al., 2010; Shaw et al., 2010; Staley and Irvine, 2010), which could mediate non-cell-autonomous function of the Hippo pathway; and the Hippo pathway genes Ex, Kibra, Crb, and Fj (Cho et al., 2006; Hamaratoglu et al., 2006; Genevet et al., 2009; Genevet et al., 2010), which may constitute a signal feedback loop.

Upstream regulators of the Hippo pathway have been intensively pursued in the past years, which led to identification of many new Hippo pathway components. Two apical cytoskeleton-binding proteins, Merlin (Mer) and Expanded (Ex) (Hamaratoglu et al., 2006), and Kibra, which interacts with Mer and Ex (Baumgartner et al., 2010; Genevet et al., 2010; Yu et al., 2010), were found to activate the Hippo pathway. Double mutations of these genes produce phenotypes closely resembling those caused by mutations of Hippo pathway components. In addition, Pez may activate the Hippo pathway by binding to Kibra (Poernbacher et al., 2012). The Fat protocadherin, a cell surface molecule, was also identified as an upstream regulator of the Hippo pathway (Bennett and Harvey, 2006; Cho et al., 2006; Silva et al., 2006; Willecke et al., 2006; Tyler and Baker, 2007). Removing one copy of $y k i$ dramatically suppressed the fat mutant overgrowth phenotype (Bennett and Harvey, 2006; Silva et al., 2006; Willecke et al., 2006), indicating that $y k i$ is an important mediator of fat function. Fat activity is regulated by binding to another protocadherin, Dachsous (Ds) (Matakatsu and Blair, 2006), and is modulated by several proteins such as the casein kinase Discs overgrown (Dco) (Feng and Irvine, 2009; Sopko et al., 2009), the Golgi-resident kinase Four-jointed (Fj) (Rogulja et al., 2008; Willecke et al., 2008; Simon et al., 2010), and the Fat/Ds-interacting protein Lowfat (Lft) (Mao et al., 2009). A LIM domain-containing protein Zyxin (Zyx) also inhibits Hippo signaling possibly by physical interaction with Wts and Dachs downstream of Fat (Rauskolb et al., 2011). dJub, another LIM domain-containing protein that physically interacts with Wts and Sav, was shown to negatively regulate Hippo signaling, although the detailed mechanism has not been delineated (Das Thakur et al., 2010). A number of cell polarity and cell adhesion proteins were also found to regulate the Hippo pathway. These include the Scribble (Scrib)-Discs large (Dlg)-Lethal giant larvae (Lgl) complex, atypical protein kinase $\mathrm{C}(\mathrm{aPKC})$, Crumbs (Crb), and Echinoid (Ed) (Chen et al., 2010a; Grzeschik et al., 2010; Ling et al., 2010; Robinson et al., 2010; Yue et al., 2012), indicating a role of cell polarity and cell adhesion in Hippo pathway regulation. Furthermore, two studies identified Tao-1, a STE20 family protein kinase as a positive regulator of the Hippo pathway, possibly by directly phosphorylating and activating the Hpo kinase (Boggiano et al., 2011; Poon et al., 2011). In addition, homodimerization was also found to regulate $\mathrm{Hpo}$ kinase activity (Jin et al., 2012). Another exciting finding is that accumulation of $\mathrm{F}$-actin in vivo by loss of actin capping proteins or expression of active formin leads to tissue over 
A

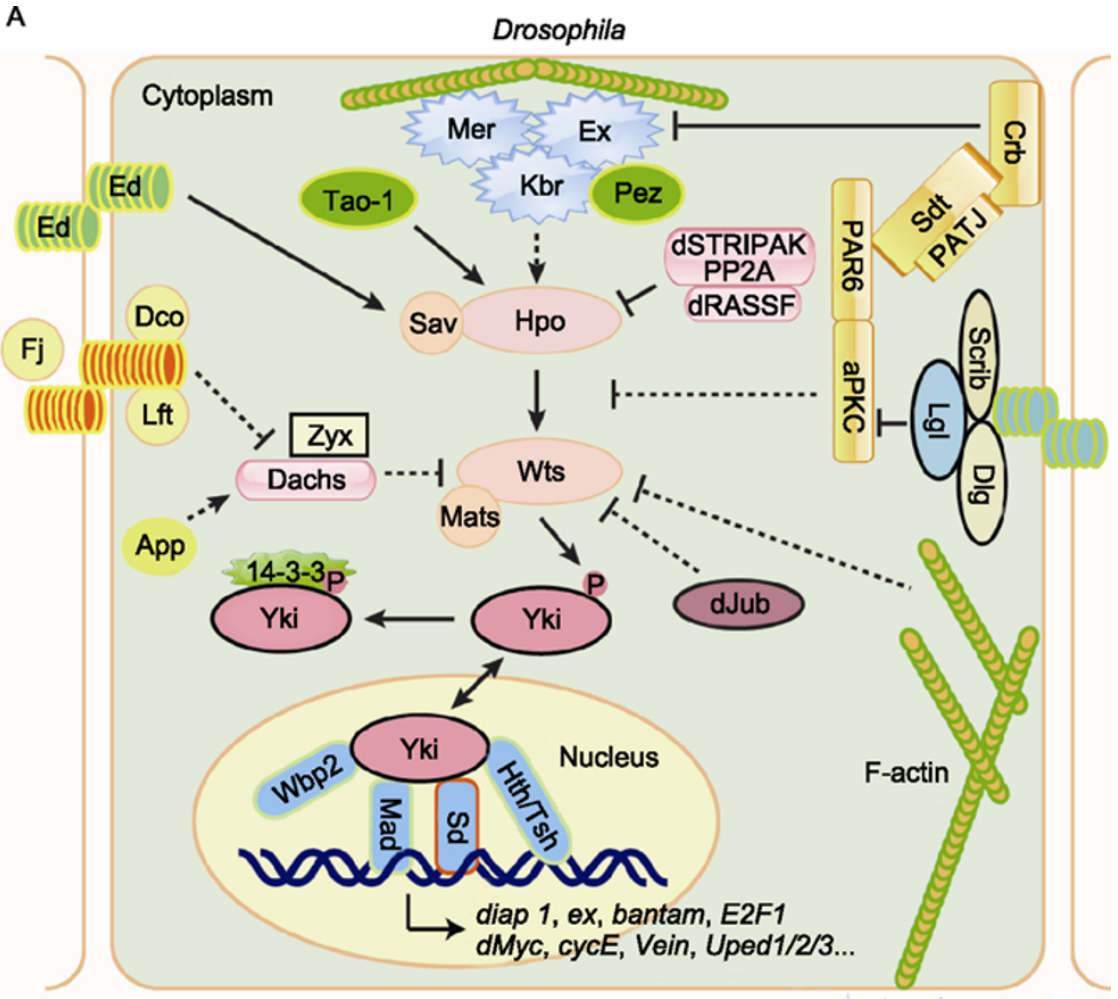

ECM

B

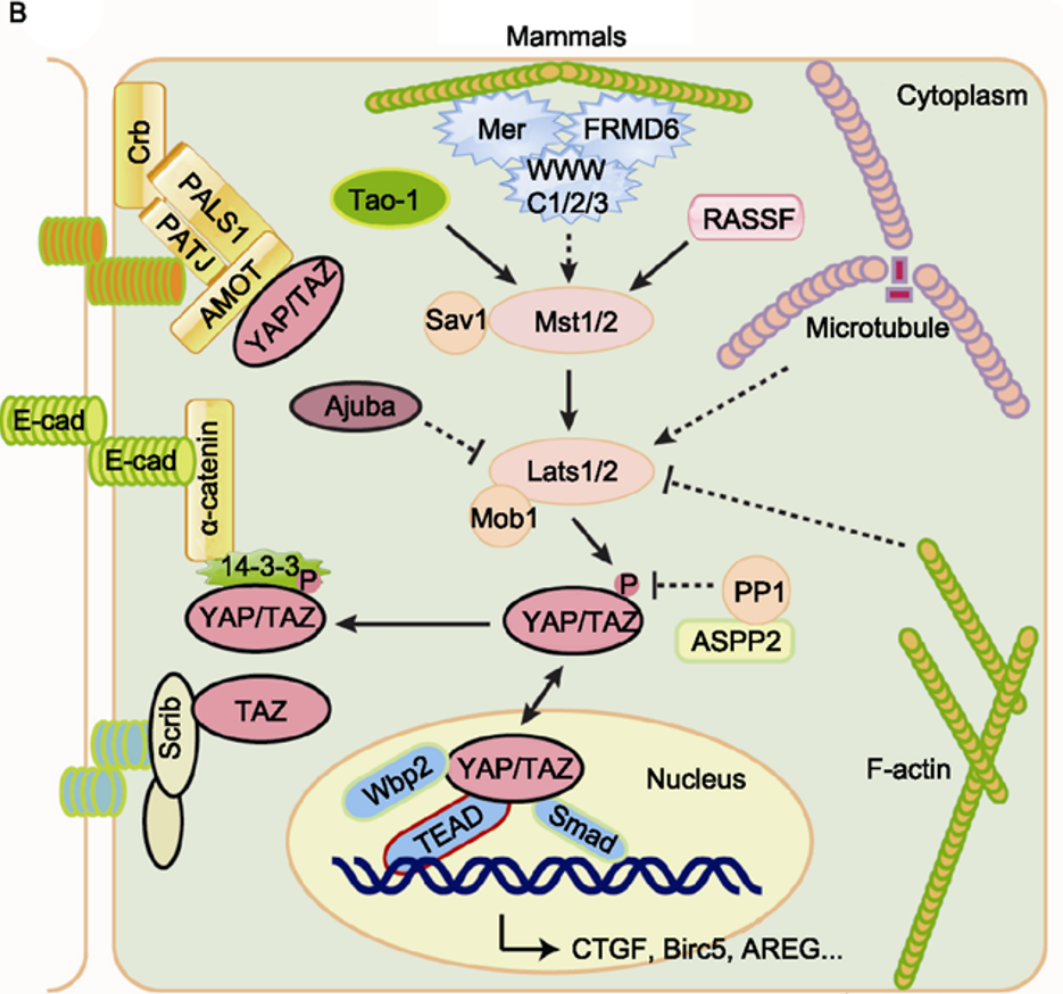

ECM

Figure 1. The Hippo pathway in Drosophila and mammals. Corresponding proteins in Drosophila (A) and mammals (B) are indicated by matching colors. Arrowed or blunted ends indicate activation or inhibition, respectively. Dashed lines indicate unknown mechanisms. 
growth due to inhibition of the Hippo pathway (Fernández et al., 2011; Sansores-Garcia et al., 2011). Such a regulation may mediate signal from epithelial architecture and external mechanical stress.

\section{THE MAMMALIAN HIPPO PATHWAY}

The core components of the Drosophila Hippo pathway are highly conserved in mammals such as Mst1/2 for Hpo, Sav1 for Sav, Lats1/2 for Wts, MOBKL1A and MOBKL1B (collectively referred to as Mob1) for Mats, and YAP and its paralog TAZ for Yki (Fig. 1B). It has been demonstrated that human YAP, Lats1, Mst2, and Mob1 can rescue the phenotypes of their corresponding Drosophila mutants in vivo (Tao et al., 1999; Wu et al., 2003; Huang et al., 2005; Lai et al., 2005), suggesting the functional conservation of these proteins. The core components Mst1/2 are known to be pro-apoptotic kinases that are activated by caspase cleavage under apoptotic stress (Graves et al., 1998). Sav1 is known to interact with Mst1/2 through the SARAH domains present in both Sav1 and Mst1/2 (Callus et al., 2006). While Sav1 has been shown to activate Mst1/2, the underlying mechanism is unclear. It has been suggested that Sav1 plays a role in the nuclear translocation of Mst1 (Lee et al., 2008). In mammalian cells, Mst1/2 are also activated by binding to Ras association domain family (RASSF) proteins (Oh et al., 2006; Guo et al., 2007) possibly due to alteration of Mst1/2 subcellular localization (Khokhlatchev et al., 2002; Praskova et al., 2004). Recently, Mst1/2 were reported to partially co-localize with actin cytoskeleton, disruption of which leads to mild activation of the kinase (Densham et al., 2009). The activation of Mst1/2 leads to phosphorylation and activation of their direct substrates, Lats1/2 (Chan et al., 2005). Mob1, which forms a complex with Lats1/2 (Chow et al., 2010), is also phosphorylated and activated by Mst1/2, resulting in enhanced interaction between Lats1/2 and Mob1 (Hirabayashi et al., 2008; Praskova et al., 2008). Similar to that in Drosophila, MST1/2/Sav1 and Lats1/2/Mob1 form a physical and functional core of the Hippo pathway. Activated Lats $1 / 2$ in turn phosphorylate YAP/TAZ transcription co-activators on several residues (Dong et al., 2007; Zhao et al., 2007; Hao et al., 2008; Lei et al., 2008; Oka et al., 2008). Phosphorylation of S127 in YAP promotes 14-3-3 binding, resulting in cytoplasmic sequestration and therefore inactivation of YAP (Dong et al., 2007; Zhao et al., 2007; Hao et al., 2008; Lei et al., 2008; Oh and Irvine, 2008). Indeed, mutation of S127 and disruption of 14-3-3 binding lead to activation of YAP (Zhao et al., 2007), confirming the inhibitory nature of this phosphorylation. YAP phosphorylation by Lats also leads to YAP inhibition through protein degradation. Phosphorylation on YAP S381 primes subsequent phosphorylation by another kinase, possibly casein kinase $1(\mathrm{CK} 1 \delta / \varepsilon)$, thereby activating a phosphorylation-dependent degradation motif termed phosphodegron. Subsequently, the activated phosphodegron recruits the E3 ubiquitin ligase $\mathrm{SCF}^{\beta-T R C P}$, leading to poly-ubiquitination and degradation of YAP (Zhao et al., 2010). This mechanism is conserved in TAZ but not in Yki (Liu et al., 2010), which lacks a residue equivalent to $S 381$.

Besides phosphorylation, other mechanisms for YAP and TAZ inhibition have been reported. YAP could interact with angiomotin (AMOT) family proteins (Varelas et al., 2010; Wang et al., 2010; Chan et al., 2011; Zhao et al., 2011), which results in YAP localization to tight junction and YAP inhibition through phosphorylation-dependent and -independent mechanisms (Zhao et al., 2011). YAP and TAZ also interact with another tight junction protein $\mathrm{ZO}-2$, which was reported to increase nuclear localization of YAP and tightjunction localization of TAZ, respectively (Oka et al., 2010; Remue et al., 2010). Interestingly, a major adherens junction protein alpha-catenin could also bind to and inhibit YAP by mediating its cell-cell junction and cytoplasmic localizations (Schlegelmilch et al., 2011; Silvis et al., 2011). Clearly, protein-protein interaction is important in the physiological regulation of YAP. Similar to that in Drosophila, the mammalian Hippo pathway is also regulated by cytoskeleton. In response to cell detachment or soft matrix, the Hippo pathway kinases Lats $1 / 2$ are activated resulting in YAP and TAZ phosphorylation and inhibition and further leading to anoikis or cell fate decision (Dupont et al., 2011; Wada et al., 2011; Zhao et al., 2012). Such regulation is largely due to the remodeling of cytoskeleton because the effect could be mimicked or blocked by interfering with actin or microtubule cytoskeleton. These studies further demonstrated the possibility of Hippo pathway as a mediator of cell shape and mechanical stress in regulation of cell physiology.

The TEAD family transcription factors were identified to be critical partners of YAP and TAZ in the regulation of gene expression (Goulev et al., 2008; Wu et al., 2008; Zhang et al., 2008; Zhao et al., 2008; Zhang et al., 2009a). Disruption of YAP-TEAD interaction abolishes YAP-dependent gene transcription and largely diminishes YAP-induced cell proliferation, oncogenic transformation, and epithelial-to-mesenchymal transition (EMT) (Zhao et al., 2008). The crystal structure of YAP-TEAD complexes reveals that the $\mathrm{N}$-terminal domain of YAP wraps around the globular structure formed by the C-terminal domain of TEAD (Chen et al., 2010b; Li et al., 2010; Tian et al., 2010). Particularly, a short peptide of YAP from residues 86 to 100 plays the most important role in YAP-TEAD interaction by fitting side chains into a deep pocket formed by TEAD. These residues could be good targets for pharmacological intervention of YAP-TEAD interaction. It is interesting to note that mutation of TEAD1 Y406, which is causal to a human genetic disease Sveinsson's chorioretinal atrophy (Fossdal et al., 2004), results in loss of interaction with YAP due to disruption of a hydrogen bond with YAP residue S94 (Kitagawa, 2007; Li et al., 2010). Precise regulation of YAP-TEAD interaction is therefore important in maintaining normal physiology. 
Connective tissue growth factor (CTGF) is the best-characterized direct target gene of YAP-TEAD. It was shown to play an important role in YAP-induced proliferation and anchorage-independent growth (Zhao et al., 2008). CTGF alone, however, does not account for all YAP phenotypes. YAP and TAZ also induce the expression of AREG (Zhang et al., 2009b) and FGF1 (Hao et al., 2008), which may also mediate non-cell-autonomous function of the Hippo pathway. However, the mechanisms underlying the induction of these genes, including the responsible transcription factors, are mostly unclear.

Functions of the Hippo pathway in organ size determination and tumor suppression have been confirmed in genetically engineered mouse models. For instance, liver-specific overexpression of YAP in transgenic mice results in enlarged liver, which is reversible upon cessation of YAP overexpression (Camargo et al., 2007; Dong et al., 2007). However, sustained YAP overexpression eventually leads to formation of tumors characteristic of hepatocellular carcinoma (HCC) (Dong et al., 2007). Furthermore, genomic amplification and elevated expression and nuclear localization of YAP has been observed in human cancers (Overholtzer et al., 2006; Zender et al., 2006; Dong et al., 2007; Zhao et al., 2007; Steinhardt et al., 2008; Hall et al., 2010). Overexpression of TAZ has also been noted in human breast cancer samples and non-small cell lung cancer cell lines (Chan et al., 2008; Zhou et al., 2011b). Similar to YAP overexpression, ablation of the Hippo pathway components Mer and Sav and double knockout of Mst1/2 in mice result in liver enlargement and tumor formation characteristic of $\mathrm{HCC}$ and cholangiocarcinoma (CC) (Zhou et al., 2009; Benhamouche et al., 2010; Lee et al., 2010; Lu et al., 2010; Song et al., 2010; Zhang et al., 2010). Aberrant Mst1/2 and Lats $1 / 2$ expression is observed in human cancers (Hisaoka et al., 2002; Jiménez-Velasco et al., 2005; Takahashi et al., 2005; Jiang et al., 2006; Minoo et al., 2007; Seidel et al., 2007; Cho et al., 2009; Zhou et al., 2009). Lats2 is also found mutated in human mesothelioma cell lines and non-small cell lung cancer (Strazisar et al., 2009; Murakami et al., 2011). Additionally, Sav1 and Mob1 mutations have been observed in human cancer cell lines and skin melanomas, respectively (Tapon et al., 2002; Lai et al., 2005). Together, these studies highlight a significant role of the Hippo pathway in organ size regulation and tumorigenesis.

\section{THE HIPPO PATHWAY LIMITS THE POOL OF TISSUE-SPECIFIC PROGENITOR CELLS}

The Hippo pathway was originally thought to regulate cell proliferation and apoptosis, thus affecting organ size. However, most organs contain not only differentiated cell types but also a pool of stem or progenitor cells. In some organs, such as pancreas, organ size is known to be regulated by the number of embryonic organ-specific progenitor cells (Stanger et al., 2007). In adults, these cells may also contribute to organ size homeostasis and regeneration after injury. Later evidence supports that in many tissues the Hippo pathway has a stronger impact on the tissue-specific stem cell compartment, possibly inhibiting their proliferation or self-renewal. It was first observed that intestinal-specific overexpression of YAP or knockout of Mst1/2 in mice caused marked expansion of the stem cell compartment (Camargo et al., 2007; Zhou et al., 2011a). Knockout of Hippo pathway components Mst1/2, Sav1, and Mer in liver also leads to accumulation of liver stem cells (Benhamouche et al., 2010; Lee et al., 2010; Lu et al., 2010; Song et al., 2010). The regulation of liver and intestinal stem cells by the Hippo pathway and its role in regeneration were discussed in great detail elsewhere (Chen et al., 2012).

The effect of the Hippo pathway on neural stem cells (NSCs) was also studied in several species. In a chicken neural tube model, overexpression of YAP markedly expands the neural progenitor cells (Cao et al., 2008). In this case, YAP clearly promotes cell proliferation in these cells as indicated by cell cycle markers. At the same time, differentiation was inhibited as shown by the attenuation of differentiation markers. The activity of YAP in neural progenitor cells depends on TEAD because a TEAD-binding-deficient form of YAP could not promote neural progenitor cell expansion. In contrast to overexpression, knockdown of YAP or introduction of a dominant-negative TEAD leads to apoptosis or differentiation of neural progenitor cells. Loss of FatJ, homolog of the Drosophila Fat protocadherin, causes similar phenotypes as YAP overexpression, and the phenotype was reversed by knockdown of YAP (Van Hateren et al., 2011). In Xenopus, overexpression or inhibition of YAP also leads to expansion or shrinkage of the pool of neural progenitors respectively (Gee et al., 2011). In Drosophila, mutation of fat or overexpression of Yki causes delay of neuroblast differentiation (Kawamori et al., 2011). Furthermore, the expression of YAP in developing mouse forebrain is restricted to the stem cell compartment (Li et al., 2012). Such a stem cell-specific expression pattern of YAP has also been observed in mouse intestine (Camargo et al., 2007). In mouse brain, YAP was also found to be a direct target of Notch intracellular domain, which provides a mechanism for Hippo pathway and Notch signaling cross-talk in NSC self-renewal (Li et al., 2012). However, the physiological signals stimulating the Hippo pathway in NSC to regulate self-renewal and proliferation are yet to be identified.

Skin is an organ constantly replenished by dividing progenitors. The role of the Hippo pathway in skin progenitors has also been studied. Similar to intestine and brain, the expression and nuclear localization of YAP is also significantly higher in the basal epidermis progenitor compartment (Schlegelmilch et al., 2011). Nuclear YAP progressively declines with age and correlates with proliferative potential of progenitors (Zhang et al., 2011). K14-Cre-driven expression of YAP caused marked expansion of basal progenitor cells 
(Schlegelmilch et al., 2011; Zhang et al., 2011). Staining of specific markers suggested that these cells were actively proliferating. Differentiation was also repressed as indicated by the expression of stem cell markers rather than those of terminal differentiation. Consistently, knockout of YAP or knock-in of a TEAD-binding-deficient form of YAP inhibits progenitor proliferation and leads to failure of skin expansion (Schlegelmilch et al., 2011). Perhaps the most interesting discovery about the Hippo pathway in skin progenitors is the identification of alpha-catenin as a direct inhibitor of YAP. Knockout of alpha-catenin clearly increases nuclear localization of YAP (Schlegelmilch et al., 2011; Silvis et al., 2011). Alpha-catenin is an adherens junction protein in association with E-cadherin and beta-catenin, and plays a key role in epithelium integrity. However, knockdown of E-cadherin and beta-catenin does not exhibit similar effect on YAP localization. It was found that alpha-catenin directly binds to YAP, which may explain the specificity (Schlegelmilch et al., 2011; Silvis et al., 2011). Knockout of alpha-catenin in bulge stem cell also leads to nuclear localized YAP and skin squamous cell carcinoma (Silvis et al., 2011). The role of YAP in alpha-catenin-mediated stem cell expansion and tumorigenesis needs to be further demonstrated by mice with double knockout of alpha-catenin and YAP. Whether the Hippo pathway still plays a role in YAP inhibition with the presence of alpha-catenin is a question not fully addressed. One study suggests that knockout of the Hippo pathway kinases Mst1/2 had no effect on skin progenitor cells (Schlegelmilch et al., 2011). However, another study of Sav1 knockout mice demonstrated a skin phenotype in embryos closely resembles that of YAP overexpression (Lee et al., 2008). Thus the role of classical Hippo pathway in restricting YAP activity in skin progenitors needs to be further clarified.

Despite the important role of the Hippo pathway in the stem cell compartment of several organs, not all tissue-specific progenitors are regulated by this pathway. One such example is the hematopoietic system. Transgenic expression of YAP caused unaltered progenitor cell compartment and no changes in the distribution of the hematopoietic lineages compared to control mice (Jansson and Larsson, 2012). Similarly, knockout of Sav1 in mouse heart leads to cardiomegaly due to abnormal proliferation of cardiomyocytes, but the number of cardiac progenitor cells remained normal (Heallen et al., 2011). Therefore, it would be important to further investigate the regulation, function, and specificity of the Hippo pathway in each organ in order to understand the role of the Hippo pathway in both differentiated cells and stem cells in organ development. Such knowledge would be a prerequisite for regenerative medicine use of the Hippo pathway in the future.

\section{THE HIPPO PATHWAY REGULATES ES CELL SELF-RENEWAL AND IPS CELL GENERATION}

It is an exciting discovery that the Hippo pathway plays im- portant roles in differentiation and expansion of tissue-specific progenitor cells. Since then, it becomes a question in the field whether the Hippo pathway could also regulate differentiation, or on the other hand self-renewal, of the more pluripotent ESCs. It was long known that YAP is highly expressed in ESCs (Ramalho-Santos et al., 2002). However, only until recently, it was demonstrated that YAP as well as its transcription factor partner TEAD promotes ES cell self-renewal. When mouse ESCs were induced to differentiate, YAP was inactivated as indicated by decreased protein level and increased phosphorylation (Lian et al., 2010; Tamm et al., 2011). However, YAP overexpression prevents mouse ESCs from differentiation even under differentiation condition such as leukemia inhibitory factor (LIF) withdrawal (Lian et al., 2010; Tamm et al., 2011). In addition, YAP knockdown leads to loss of pluripotency under a condition that would normally support stemness (Lian et al., 2010; Tamm et al., 2011). Chromatin immunoprecipitation (ChIP) experiments demonstrated that YAP-TEAD bind to promoters of many stemnesspromoting genes such as Oct4 (Lian et al., 2010; Tamm et al., 2011). Intriguingly, knockdown of TAZ in human ESCs also led to differentiation and loss of pluripotency, although YAP was intact (Varelas et al., 2008). Such a difference in TAZ and YAP requirement by human and mouse ESCs might be explained by the differential requirement of TGFbeta or bone morphogenetic protein (BMP) signaling by human and mouse ESCs respectively. TAZ was shown to promote Smad2/3 nuclear localization in response to TGFbeta signaling and YAP was demonstrated to support Smad1-dependent transcription under BMP signaling (Varelas et al., 2008; Alarcón et al., 2009). However, the mechanism responsible for specificity of YAP and TAZ in BMP and TGFbeta signaling is not known. Another report suggests the regulation of YAP by LIF, which is required by mouse but not human ESCs, which is another possible explanation for the role of YAP and TAZ in the self-renewal of human and mouse ESCs (Tamm et al., 2011).

Separation of trophectoderm (TE), which gives rise to extraembryonic tissue, from inner cell mass (ICM), where ESCs were derived from, is the first differentiation event during development. Recent genetic studies found the Hippo pathway transcription factor TEAD4 as a determinant of TE specification (Yagi et al., 2007; Nishioka et al., 2008). Further studies suggest a model that the Hippo pathway was inactivated in outer cells during blastocyst formation due to cell "crowdness," which activates YAP to drive TEAD4-dependent expression of TE-specific genes such as Cdx2 (Nishioka et al., 2009). In ICM, the Hippo pathway remains active and YAP is inhibited to prevent TE-specific gene expression. If such a model were proved to be correct, the YAP and possibly TAZ transcription co-activators would be dispensable in early ESCs during development, which needs to be demonstrated by concomitant knockout of both YAP and TAZ.

The recent development of iPSC technology is a mile stone in stem cell research. It has been demonstrated that 
expression of a defined set of transcription factors such as Oct4, Sox2, KIf4 (OSK), and Myc can reprogram differentiated adult cells into pluripotent stem cells (Takahashi and Yamanaka, 2006). Therefore the reprogramming process breaks some kind of barriers to reset cell status. By comparing somatic cells with iPSCs, the Hippo pathway gene Lats2 was found to be significantly repressed during reprogramming, and may therefore represent such a barrier (Qin et al., 2012). Indeed, knockdown of Lats2 increased efficiency of human iPSC generation about three fold without accelerating cell proliferation (Qin et al., 2012). The elevated reprogramming efficiency depends on TAZ because concomitant knockdown of TAZ blocked the effect of Lats2 knockdown. In an independent report, co-expression of YAP was also shown to improve the efficiency of human iPSC generation by OSK (Lian et al., 2010), although in a separate study, YAP did not show any significant effect when all four factors OSKM were used (Chia et al., 2010). Nevertheless, it is still clear that inhibition of the Hippo pathway would improve iPS generation although the role of TAZ and YAP in different species might be different.

\section{THE HIPPO PATHWAY DIRECTS MESENCHYMAL STEM CELL DIFFERENTIATION}

MSCs are cells originating from bone marrow that could contribute to several mesenchyme-derived tissues such as bone, cartilage, fat and muscle. These cells are not pluripotent; however, they are multipotent due to their ability to differentiate into multiple lineages. Deregulation of MSC differentiation underlies human pathological conditions, such as the decreased osteogenesis and increased bone marrow adipogenesis during aging or immobility (Verma et al., 2002). The lineage commitment of MSCs could be governed by specific transcription events in response to soluble factors. For example, activation of peroxisome proliferator activated receptors (PPARs) by rosiglitazone largely promotes adipogenesis of MSCs (Tontonoz et al., 1994). Interestingly, TAZ but not YAP was found to interact with and inhibit the activity of PPAR $\gamma$ (Hong et al., 2005). Consistently, TAZ inhibits adipogenesis of MSCs. According to this model YAP would not be able to inhibit adipogenesis due to lack of interaction with PPAR $\gamma$. This speculation awaits confirmation. TAZ also promotes osteogenesis, possibly due to activation of Runx transcription factors (Hong et al., 2005). YAP also shows potent stimulation of osteogenesis (Dupont et al., 2011). Therefore, the Hippo pathway effectors TAZ and YAP are capable of directing MSC differentiation lineages. It has not been tested whether TEAD family transcription factors play a role in TAZand YAP-mediated differentiation of MSCs. Although TEADs seem unnecessary according to the current model of TAZ and YAP in regulation of PPAR $\gamma$ and Runx activity, it has been reported that TAZ drives aberrant osteoblastic and chondrocytic differentiation of glioma stem cells in a TEAD- dependent manner (Bhat et al., 2011). Therefore, the ability of YAP and TAZ in directing MSC differentiation may not be an atypical TEAD-independent function, although again it awaits further confirmation.

More interestingly, the recent finding of YAP and TAZ being regulated by matrix stiffness and cytoskeleton may solve another mystery of MSC differentiation. A fascinating feature of MSCs is that they could differentiate into different lineages when cultured on matrixes with different stiffness mimicking their natural tissue environment (Engler et al., 2006). For example, they differentiate into adipocytes on soft matrix and osteoblasts on stiff matrix. However, the key factor regulating differentiation program in response to matrix stiffness and mechanical stress was not known until recently it was found to be YAP and TAZ. It was demonstrated that on stiff matrix these Hippo pathway transcription co-activators localize to cell nuclei (Dupont et al., 2011). However, on soft matrix, they translocate to cytoplasm. Such a regulation depends on actin cytoskeleton remodeling but was reported to be independent of the Hippo pathway (Dupont et al., 2011). Nevertheless, in another report comparing cell attachment on stiff matrix or complete detachment, the Hippo pathway kinases Lats $1 / 2$ were found to be activated by cell detachment, also in a cytoskeleton-dependent manner (Zhao et al., 2012). It is well established that Lats $1 / 2$ directly phosphorylate YAP/TAZ and trigger their cytoplasmic translocation (Zhao et al., 2007; Lei et al., 2008). Therefore it would be important to clarify whether Lats $1 / 2$ are mediating mechanical response of YAP/ TAZ, and if not, what is the mechanism underlying YAP/TAZ regulation in this context. It has been long suspected that the Hippo pathway might be regulated by cell shape and cytoskeleton due to the involvement of several cytoskeleton-associated proteins such as Mer and Ex in the pathway. The identification of YAP and TAZ in regulation of MSC differentiation in response to mechanical stress also provides an excellent platform to test these possibilities.

\section{THE HIPPO PATHWAY AND CANCER STEM CELLS}

Recent studies of neoplastic tissues have provided evidence of self-renewing, stem-like cells within at least some types of tumors, called cancer stem cells (CSCs) (Reya et al., 2001). Although CSCs represent only a small portion of cells in a tumor, they carry the ability to seed new tumors. The differences that separate CSCs from the rest of cancer cells would therefore be a good therapeutic target in order to eradicate cancer. Interestingly, by gene expression profiling, the gene expression signature of high grade, high CSC content mammary tumors was found to overlap with TAZ/YAP-induced gene expression signature, suggesting an important role of TAZ/YAP in CSCs (Cordenonsi et al., 2011). More importantly, knockdown of TAZ impairs the self-renewal of breast CSCs as indicated by comprised CSC markers, mammosphere formation and tumor seeding in vivo (Corde- 
nonsi et al., 2011). In contrast, overexpression of TAZ promotes CSC marker expression and mammosphere formation in non-CSC cancer cell populations (Cordenonsi et al., 2011). The EMT program was known to elicit stem cell properties from epithelial cells (Mani et al., 2008). And both TAZ and YAP have been shown to induce EMT in cultured mammary epithelial cells (Overholtzer et al., 2006; Lei et al., 2008). Interestingly, induction of EMT by other transcription regulators of EMT such as Twist also elevated TAZ expression (Cordenonsi et al., 2011). Therefore it was asked whether TAZ promotes CSC self-renewal by inducing EMT or vice versa. It was shown that re-expression of E-cadherin could not reverse TAZ-induced CSC self-renewal (Cordenonsi et al., 2011). However, it was unknown whether re-expression of E-cadherin completely reversed TAZ-induced EMT, especially the mesenchymal features, which might be more important to the function of TAZ in promoting CSC self-renewal. It was shown in non-epithelial glioma stem cells that TAZ is associated with the expression of mesenchymal markers and higher-grade gliomas, which are less differentiated (Bhat et al., 2011). One possibility is that TAZ is downstream of EMT-inducing transcription factors and upstream of EMT, as well as EMT-induced CSC self-renewal. To finally resolve this issue requires identification of the molecular mechanisms mediating TAZ-induced EMT and EMT-induced TAZ expression. Besides breast cancer, YAP and TEAD expression was shown to be much higher in CSC compartment of certain type of medulloblastomas (Fernandez-L et al., 2009). In addition, knockout of Hippo pathway proteins in mouse liver also induces accumulation of tumorigenic liver stem cells, which would be discussed elsewhere (Zhou etc. al., this issue, ).

\section{CONCLUSIONS AND PERSPECTIVES}

Extensive studies in the last decade have established an important role of the Hippo pathway in organ size control and tumorigenesis. Recent findings regarding functions of the Hippo pathway and its effectors YAP and TAZ in several types of stem cells further raised the significance of the pathway, and possibly complicated the situation, also. YAP and TAZ under regulation by the Hippo pathway and possibly other unknown mechanisms promotes proliferation and selfrenewal of tissue-specific progenitors and ESCs, increases iPSC generation efficiency, directs lineage commitment of MSCs during differentiation, and promotes CSC self-renewal and tumor formation and progression (Fig. 2). On the other hand, the Hippo pathway could also function in differentiated somatic cells to promote proliferation, inhibit apoptosis, and

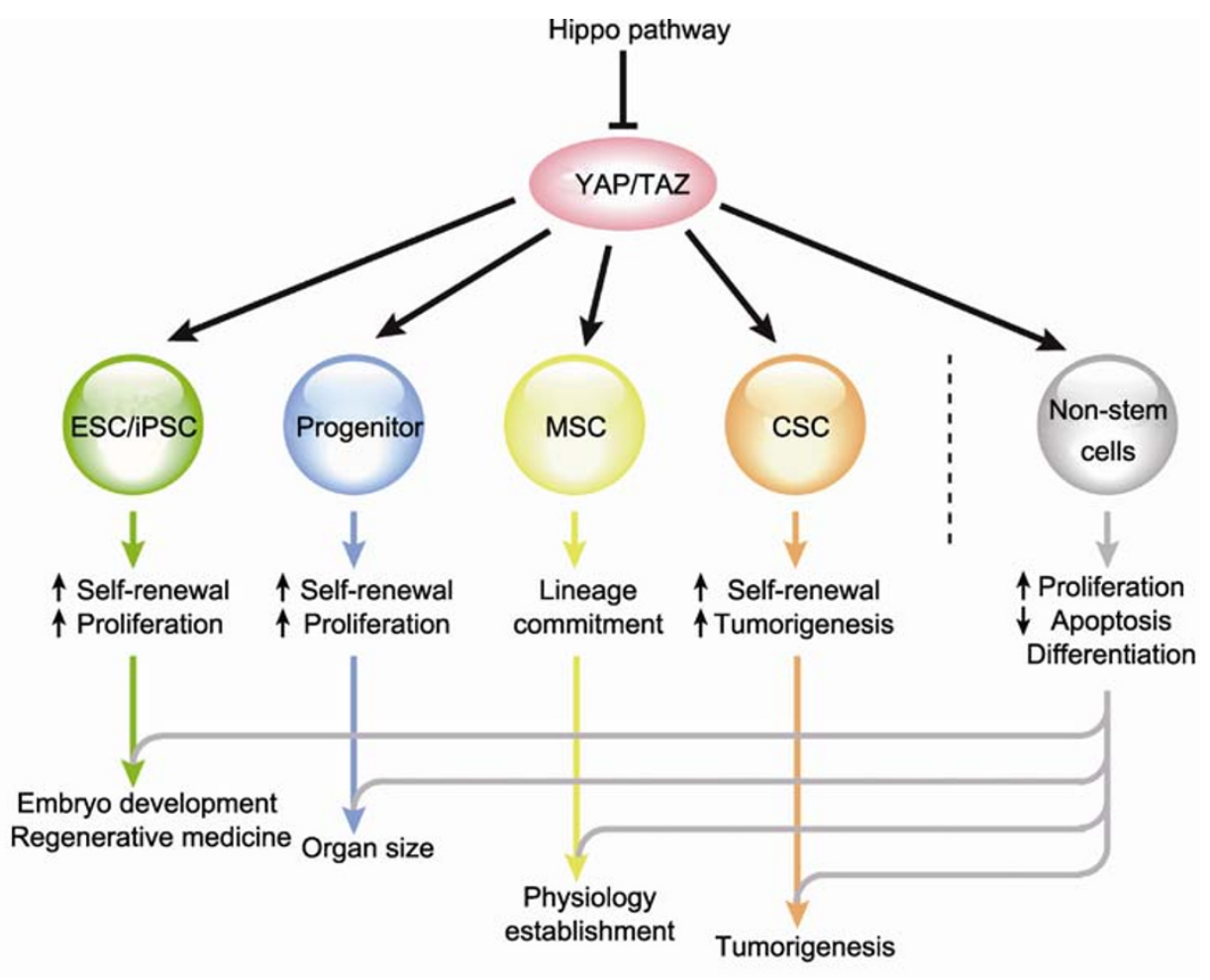

Figure 2. Function of the Hippo pathway in stem cells and differentiated cells. YAP/TAZ under regulation by the Hippo pathway functions in multiple types of stem cells as well as differentiated non-stem cells to regulate proliferation, self-renewal, differentiation, and apoptosis. Depending on cell type and organ context, the functions of the Hippo pathway in stem cells and non-stem cells coordinately control embryo development, organ size, tumorigenesis, and establishment of cell physiology. The ability of YAP/TAZ to promote reprogramming might be useful for regenerative medicine. 
regulate differentiation. These mechanisms integrate in an unclear manner to reach delicate regulation of organ size. Disturbance of the balance may result in cancer development and progression. Therefore, although the big picture seems to be on the horizon, key questions remain to be answered. Besides the questions raised above, these still include the mechanism regulating the Hippo pathway in stem cells and how is that different from the mechanisms in differentiated cells. Furthermore, the mechanism of YAP/TAZ in regulation of stem cell self-renewal should also be investigated and what is the relationship of this mechanism with that controlling cell proliferation should also be studied. Examining the cross-talk between the Hippo pathway and other signaling cascades might be important to answer these questions. In addition, it is important to understand the contributions of stem cells and differentiated cells in Hippo pathway-dependent organ size control and tumorigenesis. Eventually, it would be exciting to explore the possibility of pharmacological alteration of the Hippo pathway for cancer therapy or regenerative medicine purposes.

\section{ACKNOWLEDGEMENTS}

We apologize for those primary work that we could not cite due to space constraints. This work was supported by grants from the National Natural Science Foundation of China.

\section{ABBREVIATIONS}

BMP, bone morphogenetic protein; CSCs, cancer stem cells; CTGF, connective tissue growth factor; EMT, epithelial-to-mesenchymal transition; ESCs, embryonic stem cells; ChIP, chromatin immunoprecipitation; iPSCs, induced pluripotent stem cells; LIF, leukemia inhibitory factor; MSCs, mesenchymal stem cells; RASSF, Ras association domain family; PPARs, peroxisome proliferator activated receptors

\section{REFERENCES}

Alarcón, C., Zaromytidou, A.I., Xi, Q., Gao, S., Yu, J., Fujisawa, S., Barlas, A., Miller, A.N., Manova-Todorova, K., Macias, M.J., et al. (2009). Nuclear CDKs drive Smad transcriptional activation and turnover in BMP and TGF-beta pathways. Cell 139, 757-769.

Baumgartner, R., Poernbacher, I., Buser, N., Hafen, E., and Stocker, H. (2010). The WW domain protein Kibra acts upstream of Hippo in Drosophila. Dev Cell 18, 309-316.

Benhamouche, S., Curto, M., Saotome, I., Gladden, A.B., Liu, C.H., Giovannini, M., and McClatchey, A.I. (2010). Nf2/Merlin controls progenitor homeostasis and tumorigenesis in the liver. Genes Dev 24, 1718-1730.

Bennett, F.C., and Harvey, K.F. (2006). Fat cadherin modulates organ size in Drosophila via the Salvador/Warts/Hippo signaling pathway. Curr Biol 16, 2101-2110.
Bhat, K.P., Salazar, K.L., Balasubramaniyan, V., Wani, K., Heathcock, L., Hollingsworth, F., James, J.D., Gumin, J., Diefes, K.L., Kim, S.H., et al. (2011). The transcriptional coactivator TAZ regulates mesenchymal differentiation in malignant glioma. Genes Dev 25, 2594-2609.

Boggiano, J.C., Vanderzalm, P.J., and Fehon, R.G. (2011). Tao-1 phosphorylates Hippo/MST kinases to regulate the Hippo-Salvador-Warts tumor suppressor pathway. Dev Cell 21, 888-895.

Callus, B.A., Verhagen, A.M., and Vaux, D.L. (2006). Association of mammalian sterile twenty kinases, Mst1 and Mst2, with hSalvador via C-terminal coiled-coil domains, leads to its stabilization and phosphorylation. FEBS J 273, 4264-4276.

Camargo, F.D., Gokhale, S., Johnnidis, J.B., Fu, D., Bell, G.W., Jaenisch, R., and Brummelkamp, T.R. (2007). YAP1 increases organ size and expands undifferentiated progenitor cells. Curr Biol 17, 2054-2060.

Cao, X., Pfaff, S.L., and Gage, F.H. (2008). YAP regulates neural progenitor cell number via the TEA domain transcription factor. Genes Dev 22, 3320-3334.

Chan, E.H., Nousiainen, M., Chalamalasetty, R.B., Schäfer, A., Nigg, E.A., and Silljé, H.H. (2005). The Ste20-like kinase Mst2 activates the human large tumor suppressor kinase Lats1. Oncogene 24, 2076-2086.

Chan, S.W., Lim, C.J., Chong, Y.F., Venkatesan Pobbati, A., Huang, C., and Hong, W. (2011). Hippo pathway-independent restriction of TAZ and YAP by angiomotin. J Biol Chem 286, 7018-7026.

Chan, S.W., Lim, C.J., Guo, K., Ng, C.P., Lee, I., Hunziker, W., Zeng, Q., and Hong, W. (2008). A role for TAZ in migration, invasion, and tumorigenesis of breast cancer cells. Cancer Res 68, 2592-2598.

Chen, C.L., Gajewski, K.M., Hamaratoglu, F., Bossuyt, W., Sansores-Garcia, L., Tao, C., and Halder, G. (2010a). The apical-basal cell polarity determinant Crumbs regulates Hippo signaling in Drosophila. Proc Natl Acad Sci U S A 107, 15810-15815.

Chen, L., Chan, S.W., Zhang, X., Walsh, M., Lim, C.J., Hong, W., and Song, H. (2010b). Structural basis of YAP recognition by TEAD4 in the hippo pathway. Genes Dev 24, 290-300.

Chen, L.F., Qin, F.N., Deng, X.M., Avruch, J., Zhou, D.W. (2012). Hippo pathway in intestinal homeostasis and tumorigenesis. Protein Cell 2012 April. doi: 10.1007/s13238-012-2913-9.

Chia, N.Y., Chan, Y.S., Feng, B., Lu, X., Orlov, Y.L., Moreau, D., Kumar, P., Yang, L., Jiang, J., Lau, M.S., et al. (2010). A genome-wide RNAi screen reveals determinants of human embryonic stem cell identity. Nature 468, 316-320.

Cho, E., Feng, Y., Rauskolb, C., Maitra, S., Fehon, R., and Irvine, K.D. (2006). Delineation of a Fat tumor suppressor pathway. Nat Genet 38, 1142-1150.

Cho, W.J., Shin, J.M., Kim, J.S., Lee, M.R., Hong, K.S., Lee, J.H., Koo, K.H., Park, J.W., and Kim, K.S. (2009). miR-372 regulates cell cycle and apoptosis of ags human gastric cancer cell line through direct regulation of LATS2. Mol Cells 28, 521-527.

Chow, A., Hao, Y., and Yang, X. (2010). Molecular characterization of 
human homologs of yeast MOB1. Int J Cancer 126, 2079-2089.

Cordenonsi, M., Zanconato, F., Azzolin, L., Forcato, M., Rosato, A., Frasson, C., Inui, M., Montagner, M., Parenti, A.R., Poletti, A., et al. (2011). The Hippo transducer TAZ confers cancer stem cell-related traits on breast cancer cells. Cell 147, 759-772.

Das Thakur, M., Feng, Y., Jagannathan, R., Seppa, M.J., Skeath, J.B., and Longmore, G.D. (2010). Ajuba LIM proteins are negative regulators of the Hippo signaling pathway. Curr Biol 20, 657-662.

Densham, R.M., O'Neill, E., Munro, J., König, I., Anderson, K., Kolch, W., and Olson, M.F. (2009). MST kinases monitor actin cytoskeletal integrity and signal via c-Jun $\mathrm{N}$-terminal kinase stress-activated kinase to regulate p21Waf1/Cip1 stability. Mol Cell Biol 29, 6380-6390.

Dong, J., Feldmann, G., Huang, J., Wu, S., Zhang, N., Comerford, S.A., Gayyed, M.F., Anders, R.A., Maitra, A., and Pan, D. (2007). Elucidation of a universal size-control mechanism in Drosophila and mammals. Cell 130, 1120-1133.

Dupont, S., Morsut, L., Aragona, M., Enzo, E., Giulitti, S., Cordenonsi, M., Zanconato, F., Le Digabel, J., Forcato, M., Bicciato, S., et al. (2011). Role of YAP/TAZ in mechanotransduction. Nature 474, 179-183.

Engler, A.J., Sen, S., Sweeney, H.L., and Discher, D.E. (2006). Matrix elasticity directs stem cell lineage specification. Cell 126, 677-689.

Feng, Y., and Irvine, K.D. (2009). Processing and phosphorylation of the Fat receptor. Proc Natl Acad Sci U S A 106, 11989-11994.

Fernández, B.G., Gaspar, P., Brás-Pereira, C., Jezowska, B., Rebelo, S.R., and Janody, F. (2011). Actin-Capping Protein and the Hippo pathway regulate F-actin and tissue growth in Drosophila. Development 138, 2337-2346.

Fernandez-L, A., Northcott, P.A., Dalton, J., Fraga, C., Ellison, D., Angers, S., Taylor, M.D., and Kenney, A.M. (2009). YAP1 is amplified and up-regulated in hedgehog-associated medulloblastomas and mediates Sonic hedgehog-driven neural precursor proliferation. Genes Dev 23, 2729-2741.

Fossdal, R., Jonasson, F., Kristjansdottir, G.T., Kong, A., Stefansson, H., Gosh, S., Gulcher, J.R., and Stefansson, K. (2004). A novel TEAD1 mutation is the causative allele in Sveinsson's chorioretinal atrophy (helicoid peripapillary chorioretinal degeneration). Hum Mol Genet 13, 975-981.

Gee, S.T., Milgram, S.L., Kramer, K.L., Conlon, F.L., and Moody, S.A. (2011). Yes-associated protein 65 (YAP) expands neural progenitors and regulates $\mathrm{Pax} 3$ expression in the neural plate border zone. PLoS One 6, e20309.

Genevet, A., Polesello, C., Blight, K., Robertson, F., Collinson, L.M., Pichaud, F., and Tapon, N. (2009). The Hippo pathway regulates apical-domain size independently of its growth-control function. J Cell Sci 122, 2360-2370.

Genevet, A., Wehr, M.C., Brain, R., Thompson, B.J., and Tapon, N. (2010). Kibra is a regulator of the Salvador/Warts/Hippo signaling network. Dev Cell 18, 300-308.

Goulev, Y., Fauny, J.D., Gonzalez-Marti, B., Flagiello, D., Silber, J., and Zider, A. (2008). SCALLOPED interacts with YORKIE, the nuclear effector of the hippo tumor-suppressor pathway in Dro- sophila. Curr Biol 18, 435-441.

Graves, J.D., Gotoh, Y., Draves, K.E., Ambrose, D., Han, D.K., Wright, M., Chernoff, J., Clark, E.A., and Krebs, E.G. (1998). Caspase-mediated activation and induction of apoptosis by the mammalian Ste20-like kinase Mst1. EMBO J 17, 2224-2234.

Grzeschik, N.A., Parsons, L.M., Allott, M.L., Harvey, K.F., and Richardson, H.E. (2010). Lgl, aPKC, and Crumbs regulate the Salvador/Warts/Hippo pathway through two distinct mechanisms. Curr Biol 20, 573-581.

Guo, C., Tommasi, S., Liu, L., Yee, J.K., Dammann, R., and Pfeifer, G.P. (2007). RASSF1A is part of a complex similar to the Drosophila Hippo/Salvador/Lats tumor-suppressor network. Curr Biol 17, 700-705.

Hall, C.A., Wang, R., Miao, J., Oliva, E., Shen, X., Wheeler, T., Hilsenbeck, S.G., Orsulic, S., and Goode, S. (2010). Hippo pathway effector Yap is an ovarian cancer oncogene. Cancer Res 70, 8517-8525.

Hamaratoglu, F., Willecke, M., Kango-Singh, M., Nolo, R., Hyun, E., Tao, C., Jafar-Nejad, H., and Halder, G. (2006). The tumour-suppressor genes NF2/Merlin and Expanded act through Hippo signalling to regulate cell proliferation and apoptosis. Nat Cell Biol 8, 27-36.

Hao, Y., Chun, A., Cheung, K., Rashidi, B., and Yang, X. (2008). Tumor suppressor LATS1 is a negative regulator of oncogene YAP. J Biol Chem 283, 5496-5509.

Hariharan, I.K., and Bilder, D. (2006). Regulation of imaginal disc growth by tumor-suppressor genes in Drosophila. Annu Rev Genet 40, 335-361.

Harvey, K.F., Pfleger, C.M., and Hariharan, I.K. (2003). The Drosophila Mst ortholog, hippo, restricts growth and cell proliferation and promotes apoptosis. Cell 114, 457-467.

Heallen, T., Zhang, M., Wang, J., Bonilla-Claudio, M., Klysik, E., Johnson, R.L., and Martin, J.F. (2011). Hippo pathway inhibits Wnt signaling to restrain cardiomyocyte proliferation and heart size. Science 332, 458-461.

Hirabayashi, S., Nakagawa, K., Sumita, K., Hidaka, S., Kawai, T., Ikeda, M., Kawata, A., Ohno, K., and Hata, Y. (2008). Threonine 74 of MOB1 is a putative key phosphorylation site by MST2 to form the scaffold to activate nuclear Dbf2-related kinase 1. Oncogene 27, 4281-4292.

Hisaoka, M., Tanaka, A., and Hashimoto, H. (2002). Molecular alterations of h-warts/LATS1 tumor suppressor in human soft tissue sarcoma. Lab Invest 82, 1427-1435.

Hong, J.H., Hwang, E.S., McManus, M.T., Amsterdam, A., Tian, Y., Kalmukova, R., Mueller, E., Benjamin, T., Spiegelman, B.M., Sharp, P.A., et al. (2005). TAZ, a transcriptional modulator of mesenchymal stem cell differentiation. Science 309, 1074-1078.

Huang, J., Wu, S., Barrera, J., Matthews, K., and Pan, D. (2005). The Hippo signaling pathway coordinately regulates cell proliferation and apoptosis by inactivating Yorkie, the Drosophila Homolog of YAP. Cell 122, 421-434.

Jansson, L., and Larsson, J. (2012). Normal Hematopoietic Stem Cell Function in Mice with Enforced Expression of the Hippo Signaling Effector YAP1. PLoS One 7, e32013. 
Jia, J., Zhang, W., Wang, B., Trinko, R., and Jiang, J. (2003). The Drosophila Ste20 family kinase dMST functions as a tumor suppressor by restricting cell proliferation and promoting apoptosis. Genes Dev 17, 2514-2519.

Jiang, Z., Li, X., Hu, J., Zhou, W., Jiang, Y., Li, G., and Lu, D. (2006). Promoter hypermethylation-mediated down-regulation of LATS1 and LATS2 in human astrocytoma. Neurosci Res 56, 450-458.

Jiménez-Velasco, A., Román-Gómez, J., Agirre, X., Barrios, M., Navarro, G., Vázquez, I., Prósper, F., Torres, A., and Heiniger, A. (2005). Downregulation of the large tumor suppressor 2 (LATS2/KPM) gene is associated with poor prognosis in acute lymphoblastic leukemia. Leukemia 19, 2347-2350.

Jin, Y., Dong, L., Lu, Y., Wu, W., Hao, Q., Zhou, Z., Jiang, J., Zhao, Y., and Zhang, L. (2012). Dimerization and cytoplasmic localization regulate hippo kinase signaling activity in organ size control. J Biol Chem 287, 5784-5796.

Justice, R.W., Zilian, O., Woods, D.F., Noll, M., and Bryant, P.J. (1995). The Drosophila tumor suppressor gene warts encodes a homolog of human myotonic dystrophy kinase and is required for the control of cell shape and proliferation. Genes Dev 9, 534-546.

Kango-Singh, M., Nolo, R., Tao, C., Verstreken, P., Hiesinger, P.R., Bellen, H.J., and Halder, G. (2002). Shar-pei mediates cell proliferation arrest during imaginal disc growth in Drosophila. Development 129, 5719-5730.

Karpowicz, P., Perez, J., and Perrimon, N. (2010). The Hippo tumor suppressor pathway regulates intestinal stem cell regeneration. Development 137, 4135-4145.

Kawamori, H., Tai, M., Sato, M., Yasugi, T., and Tabata, T. (2011). Fat/Hippo pathway regulates the progress of neural differentiation signaling in the Drosophila optic lobe. Dev Growth Differ 53, 653-667.

Khokhlatchev, A., Rabizadeh, S., Xavier, R., Nedwidek, M., Chen, T., Zhang, X.F., Seed, B., and Avruch, J. (2002). Identification of a novel Ras-regulated proapoptotic pathway. Curr Biol 12, 253-265.

Kitagawa, M. (2007). A Sveinsson's chorioretinal atrophy-associated missense mutation in mouse Tead1 affects its interaction with the co-factors YAP and TAZ. Biochem Biophys Res Commun 361, 1022-1026.

Lai, Z.C., Wei, X., Shimizu, T., Ramos, E., Rohrbaugh, M., Nikolaidis, N., Ho, L.L., and Li, Y. (2005). Control of cell proliferation and apoptosis by mob as tumor suppressor, mats. Cell 120, 675-685.

Lee, J.H., Kim, T.S., Yang, T.H., Koo, B.K., Oh, S.P., Lee, K.P., Oh, H.J., Lee, S.H., Kong, Y.Y., Kim, J.M., et al. (2008). A crucial role of WW45 in developing epithelial tissues in the mouse. EMBO J 27, 1231-1242.

Lee, K.P., Lee, J.H., Kim, T.S., Kim, T.H., Park, H.D., Byun, J.S., Kim, M.C., Jeong, W.I., Calvisi, D.F., Kim, J.M., et al. (2010). The Hippo-Salvador pathway restrains hepatic oval cell proliferation, liver size, and liver tumorigenesis. Proc Natl Acad Sci U S A 107, 8248-8253.

Lei, Q.Y., Zhang, H., Zhao, B., Zha, Z.Y., Bai, F., Pei, X.H., Zhao, S., Xiong, Y., and Guan, K.L. (2008). TAZ promotes cell proliferation and epithelial-mesenchymal transition and is inhibited by the hippo pathway. Mol Cell Biol 28, 2426-2436.
Li, Y., Hibbs, M.A., Gard, A.L., Shylo, N.A., and Yun, K. (2012). Genome-wide analysis of N1ICD/RBPJ targets in vivo reveals direct transcriptional regulation of $\mathrm{Wnt}, \mathrm{SHH}$, and Hippo pathway effectors by Notch1. Stem Cells 2012 Jan 9. doi: 10.1002/stem.1030. [Epub ahead of print]

Li, Z., Zhao, B., Wang, P., Chen, F., Dong, Z., Yang, H., Guan, K.L., and $\mathrm{Xu}, \mathrm{Y}$. (2010). Structural insights into the YAP and TEAD complex. Genes Dev 24, 235-240.

Lian, I., Kim, J., Okazawa, H., Zhao, J., Zhao, B., Yu, J., Chinnaiyan, A., Israel, M.A., Goldstein, L.S., Abujarour, R., et al. (2010). The role of YAP transcription coactivator in regulating stem cell self-renewal and differentiation. Genes Dev 24, 1106-1118.

Ling, C., Zheng, Y., Yin, F., Yu, J., Huang, J., Hong, Y., Wu, S., and Pan, D. (2010). The apical transmembrane protein Crumbs functions as a tumor suppressor that regulates Hippo signaling by binding to Expanded. Proc Natl Acad Sci U S A 107, 10532-10537.

Liu, C.Y., Zha, Z.Y., Zhou, X., Zhang, H., Huang, W., Zhao, D., Li, T., Chan, S.W., Lim, C.J., Hong, W., et al. (2010). The hippo tumor pathway promotes TAZ degradation by phosphorylating a phosphodegron and recruiting the SCFbeta-TrCP E3 ligase. J Biol Chem 285, 37159-37169.

Lu, L., Li, Y., Kim, S.M., Bossuyt, W., Liu, P., Qiu, Q., Wang, Y., Halder, G., Finegold, M.J., Lee, J.S., et al. (2010). Hippo signaling is a potent in vivo growth and tumor suppressor pathway in the mammalian liver. Proc Natl Acad Sci U S A 107, 1437-1442.

Mani, S.A., Guo, W., Liao, M.J., Eaton, E.N., Ayyanan, A., Zhou, A.Y., Brooks, M., Reinhard, F., Zhang, C.C., Shipitsin, M., et al. (2008). The epithelial-mesenchymal transition generates cells with properties of stem cells. Cell 133, 704-715.

Mao, Y., Kucuk, B., and Irvine, K.D. (2009). Drosophila lowfat, a novel modulator of Fat signaling. Development 136, 3223-3233.

Matakatsu, H., and Blair, S.S. (2006). Separating the adhesive and signaling functions of the Fat and Dachsous protocadherins. Development 133, 2315-2324.

Minoo, P., Zlobec, I., Baker, K., Tornillo, L., Terracciano, L., Jass, J.R., and Lugli, A. (2007). Prognostic significance of mammalian sterile20-like kinase 1 in colorectal cancer. Mod Pathol 20, 331-338.

Murakami, H., Mizuno, T., Taniguchi, T., Fujii, M., Ishiguro, F., Fukui, T., Akatsuka, S., Horio, Y., Hida, T., Kondo, Y., et al. (2011). LATS2 is a tumor suppressor gene of malignant mesothelioma. Cancer Res 71, 873-883.

Neto-Silva, R.M., de Beco, S., and Johnston, L.A. (2010). Evidence for a growth-stabilizing regulatory feedback mechanism between Myc and Yorkie, the Drosophila homolog of Yap. Dev Cell 19, 507-520.

Nishioka, N., Inoue, K., Adachi, K., Kiyonari, H., Ota, M., Ralston, A., Yabuta, N., Hirahara, S., Stephenson, R.O., Ogonuki, N., et al. (2009). The Hippo signaling pathway components Lats and Yap pattern Tead4 activity to distinguish mouse trophectoderm from inner cell mass. Dev Cell 16, 398-410.

Nishioka, N., Yamamoto, S., Kiyonari, H., Sato, H., Sawada, A., Ota, M., Nakao, K., and Sasaki, H. (2008). Tead4 is required for speci- 
fication of trophectoderm in pre-implantation mouse embryos. Mech Dev 125, 270-283.

Oh, H., and Irvine, K.D. (2008). In vivo regulation of Yorkie phosphorylation and localization. Development 135, 1081-1088.

Oh, H.J., Lee, K.K., Song, S.J., Jin, M.S., Song, M.S., Lee, J.H., Im, C.R., Lee, J.O., Yonehara, S., and Lim, D.S. (2006). Role of the tumor suppressor RASSF1A in Mst1-mediated apoptosis. Cancer Res 66, 2562-2569.

Oka, T., Mazack, V., and Sudol, M. (2008). Mst2 and Lats kinases regulate apoptotic function of Yes kinase-associated protein (YAP). J Biol Chem 283, 27534-27546.

Oka, T., Remue, E., Meerschaert, K., Vanloo, B., Boucherie, C., Gfeller, D., Bader, G.D., Sidhu, S.S., Vandekerckhove, J., Gettemans, J., et al. (2010). Functional complexes between YAP2 and ZO-2 are PDZ domain-dependent, and regulate YAP2 nuclear localization and signalling. Biochem J 432, 461-472.

Overholtzer, M., Zhang, J., Smolen, G.A., Muir, B., Li, W., Sgroi, D.C., Deng, C.X., Brugge, J.S., and Haber, D.A. (2006). Transforming properties of YAP, a candidate oncogene on the chromosome 11q22 amplicon. Proc Natl Acad Sci U S A 103, 12405-12410.

Pan, D. (2010). The hippo signaling pathway in development and cancer. Dev Cell 19, 491-505.

Pantalacci, S., Tapon, N., and Léopold, P. (2003). The Salvador partner Hippo promotes apoptosis and cell-cycle exit in Drosophila. Nat Cell Biol 5, 921-927.

Poernbacher, I., Baumgartner, R., Marada, S.K., Edwards, K., and Stocker, H. (2012). Drosophila pez acts in hippo signaling to restrict intestinal stem cell proliferation. Curr Biol 22, 389-396.

Poon, C.L., Lin, J.I., Zhang, X., and Harvey, K.F. (2011). The sterile 20-like kinase Tao-1 controls tissue growth by regulating the Salvador-Warts-Hippo pathway. Dev Cell 21, 896-906.

Praskova, M., Khoklatchev, A., Ortiz-Vega, S., and Avruch, J. (2004). Regulation of the MST1 kinase by autophosphorylation, by the growth inhibitory proteins, RASSF1 and NORE1, and by Ras. Biochem J 381, 453-462.

Praskova, M., Xia, F., and Avruch, J. (2008). MOBKL1A/MOBKL1B phosphorylation by MST1 and MST2 inhibits cell proliferation. Curr Biol 18, 311-321.

Qin, H., Blaschke, K., Wei, G., Ohi, Y., Blouin, L., Qi, Z., Yu, J., Yeh, R.F., Hebrok, M., and Ramalho-Santos, M. (2012). Transcriptional analysis of pluripotency reveals the Hippo pathway as a barrier to reprogramming. Human Mol Genet Jan 27, 2012. doi: 10.1093/hmg/dds023. [published online]

Ramalho-Santos, M., Yoon, S., Matsuzaki, Y., Mulligan, R.C., and Melton, D.A. (2002). "Stemness": transcriptional profiling of embryonic and adult stem cells. Science 298, 597-600.

Rauskolb, C., Pan, G., Reddy, B.V., Oh, H., and Irvine, K.D. (2011). Zyxin links fat signaling to the hippo pathway. PLoS Biol 9, e1000624.

Remue, E., Meerschaert, K., Oka, T., Boucherie, C., Vandekerckhove, J., Sudol, M., and Gettemans, J. (2010). TAZ interacts with zonula occludens-1 and -2 proteins in a PDZ-1 dependent manner. FEBS Lett 584, 4175-4180.

Ren, F., Wang, B., Yue, T., Yun, E.Y., Ip, Y.T., and Jiang, J. (2010).
Hippo signaling regulates Drosophila intestine stem cell proliferation through multiple pathways. Proc Natl Acad Sci U S A 107, 21064-21069.

Reya, T., Morrison, S.J., Clarke, M.F., and Weissman, I.L. (2001). Stem cells, cancer, and cancer stem cells. Nature 414, 105-111.

Ribeiro, P.S., Josué, F., Wepf, A., Wehr, M.C., Rinner, O., Kelly, G., Tapon, N., and Gstaiger, M. (2010). Combined functional genomic and proteomic approaches identify a PP2A complex as a negative regulator of Hippo signaling. Mol Cell 39, 521-534.

Robinson, B.S., Huang, J., Hong, Y., and Moberg, K.H. (2010). Crumbs regulates Salvador/Warts/Hippo signaling in Drosophila via the FERM-domain protein Expanded. Curr Biol 20, 582-590.

Rogulja, D., Rauskolb, C., and Irvine, K.D. (2008). Morphogen control of wing growth through the Fat signaling pathway. Dev Cell 15, 309-321.

Sansores-Garcia, L., Bossuyt, W., Wada, K., Yonemura, S., Tao, C., Sasaki, H., and Halder, G. (2011). Modulating F-actin organization induces organ growth by affecting the Hippo pathway. EMBO J 30 , 2325-2335.

Schlegelmilch, K., Mohseni, M., Kirak, O., Pruszak, J., Rodriguez, J.R., Zhou, D., Kreger, B.T., Vasioukhin, V., Avruch, J., Brummelkamp, T.R., et al. (2011). Yap1 acts downstream of -catenin to control epidermal proliferation. Cell 144, 782-795.

Seidel, C., Schagdarsurengin, U., Blümke, K., Würl, P., Pfeifer, G.P., Hauptmann, S., Taubert, H., and Dammann, R. (2007). Frequent hypermethylation of MST1 and MST2 in soft tissue sarcoma. Mol Carcinog 46, 865-871.

Shaw, R.L., Kohlmaier, A., Polesello, C., Veelken, C., Edgar, B.A., and Tapon, N. (2010). The Hippo pathway regulates intestinal stem cell proliferation during Drosophila adult midgut regeneration. Development 137, 4147-4158.

Silva, E., Tsatskis, Y., Gardano, L., Tapon, N., and McNeill, H. (2006). The tumor-suppressor gene fat controls tissue growth upstream of expanded in the hippo signaling pathway. Curr Biol 16, 2081-2089.

Silvis, M.R., Kreger, B.T., Lien, W.H., Klezovitch, O., Rudakova, G.M., Camargo, F.D., Lantz, D.M., Seykora, J.T., and Vasioukhin, V. (2011). alpha-catenin is a tumor suppressor that controls cell accumulation by regulating the localization and activity of the transcriptional coactivator Yap1. Sci Signal 4, ra33.

Simon, M.A., Xu, A., Ishikawa, H.O., and Irvine, K.D. (2010). Modulation of fat:dachsous binding by the cadherin domain kinase four-jointed. Curr Biol 20, 811-817.

Song, H., Mak, K.K., Topol, L., Yun, K., Hu, J., Garrett, L., Chen, Y., Park, O., Chang, J., Simpson, R.M., et al. (2010). Mammalian Mst1 and Mst2 kinases play essential roles in organ size control and tumor suppression. Proc Natl Acad Sci U S A 107, 1431-1436.

Sopko, R., Silva, E., Clayton, L., Gardano, L., Barrios-Rodiles, M., Wrana, J., Varelas, X., Arbouzova, N.I., Shaw, S., Saburi, S., et al. (2009). Phosphorylation of the tumor suppressor fat is regulated by its ligand Dachsous and the kinase discs overgrown. Curr Biol 19, 1112-1117.

Staley, B.K., and Irvine, K.D. (2010). Warts and Yorkie mediate in- 
testinal regeneration by influencing stem cell proliferation. Curr Biol 20, 1580-1587.

Stanger, B.Z., Tanaka, A.J., and Melton, D.A. (2007). Organ size is limited by the number of embryonic progenitor cells in the pancreas but not the liver. Nature 445, 886-891.

Steinhardt, A.A., Gayyed, M.F., Klein, A.P., Dong, J., Maitra, A., Pan, D., Montgomery, E.A., and Anders, R.A. (2008). Expression of Yes-associated protein in common solid tumors. Hum Pathol 39, 1582-1589.

Strazisar, M., Mlakar, V., and Glavac, D. (2009). LATS2 tumour specific mutations and down-regulation of the gene in non-small cell carcinoma. Lung Cancer 64, 257-262.

Takahashi, K., and Yamanaka, S. (2006). Induction of pluripotent stem cells from mouse embryonic and adult fibroblast cultures by defined factors. Cell 126, 663-676.

Takahashi, Y., Miyoshi, Y., Takahata, C., Irahara, N., Taguchi, T., Tamaki, Y., and Noguchi, S. (2005). Down-regulation of LATS1 and LATS2 mRNA expression by promoter hypermethylation and its association with biologically aggressive phenotype in human breast cancers. Clin Cancer Res 11, 1380-1385.

Tamm, C., Böwer, N., and Annerén, C. (2011). Regulation of mouse embryonic stem cell self-renewal by a Yes-YAP-TEAD2 signaling pathway downstream of LIF. J Cell Sci 124, 1136-1144.

Tao, W., Zhang, S., Turenchalk, G.S., Stewart, R.A., St John, M.A., Chen, W., and Xu, T. (1999). Human homologue of the Drosophila melanogaster lats tumour suppressor modulates CDC2 activity. Nat Genet 21, 177-181.

Tapon, N., Harvey, K.F., Bell, D.W., Wahrer, D.C., Schiripo, T.A., Haber, D.A., and Hariharan, I.K. (2002). salvador Promotes both cell cycle exit and apoptosis in Drosophila and is mutated in human cancer cell lines. Cell 110, 467-478.

Tian, W., Yu, J., Tomchick, D.R., Pan, D., and Luo, X. (2010). Structural and functional analysis of the YAP-binding domain of human TEAD2. Proc Natl Acad Sci U S A 107, 7293-7298.

Tontonoz, P., Hu, E., and Spiegelman, B.M. (1994). Stimulation of adipogenesis in fibroblasts by PPAR gamma 2, a lipid-activated transcription factor. Cell 79, 1147-1156.

Tyler, D.M., and Baker, N.E. (2007). Expanded and fat regulate growth and differentiation in the Drosophila eye through multiple signaling pathways. Dev Biol 305, 187-201.

Udan, R.S., Kango-Singh, M., Nolo, R., Tao, C., and Halder, G. (2003). Hippo promotes proliferation arrest and apoptosis in the Salvador/Warts pathway. Nat Cell Biol 5, 914-920.

Van Hateren, N.J., Das, R.M., Hautbergue, G.M., Borycki, A.G., Placzek, M., and Wilson, S.A. (2011). FatJ acts via the Hippo mediator Yap1 to restrict the size of neural progenitor cell pools. Development 138, 1893-1902.

Varelas, X., Sakuma, R., Samavarchi-Tehrani, P., Peerani, R., Rao, B.M., Dembowy, J., Yaffe, M.B., Zandstra, P.W., and Wrana, J.L. (2008). TAZ controls Smad nucleocytoplasmic shuttling and regulates human embryonic stem-cell self-renewal. Nat Cell Biol 10, 837-848.

Varelas, X., Samavarchi-Tehrani, P., Narimatsu, M., Weiss, A., Cockburn, K., Larsen, B.G., Rossant, J., and Wrana, J.L. (2010).
The Crumbs complex couples cell density sensing to Hippo-dependent control of the TGF- $\beta-S M A D$ pathway. Dev Cell 19, 831-844.

Verma, S., Rajaratnam, J.H., Denton, J., Hoyland, J.A., and Byers, R.J. (2002). Adipocytic proportion of bone marrow is inversely related to bone formation in osteoporosis. J Clin Pathol 55, 693-698.

Wada, K., Itoga, K., Okano, T., Yonemura, S., and Sasaki, H. (2011). Hippo pathway regulation by cell morphology and stress fibers. Development 138, 3907-3914.

Wang, W., Huang, J., and Chen, J. (2010). Angiomotin-like proteins associate with and negatively regulate YAP1. J Biol Chem J Biol Chem 286, 4364-4370.

Wei, X., Shimizu, T., and Lai, Z.C. (2007). Mob as tumor suppressor is activated by Hippo kinase for growth inhibition in Drosophila. EMBO J 26, 1772-1781.

Willecke, M., Hamaratoglu, F., Kango-Singh, M., Udan, R., Chen, C.L., Tao, C., Zhang, X., and Halder, G. (2006). The fat cadherin acts through the hippo tumor-suppressor pathway to regulate tissue size. Curr Biol 16, 2090-2100.

Willecke, M., Hamaratoglu, F., Sansores-Garcia, L., Tao, C., and Halder, G. (2008). Boundaries of Dachsous Cadherin activity modulate the Hippo signaling pathway to induce cell proliferation. Proc Natl Acad Sci U S A 105, 14897-14902.

Wu, S., Huang, J., Dong, J., and Pan, D. (2003). hippo encodes a Ste-20 family protein kinase that restricts cell proliferation and promotes apoptosis in conjunction with salvador and warts. Cell 114, 445-456.

Wu, S., Liu, Y., Zheng, Y., Dong, J., and Pan, D. (2008). The TEAD/TEF family protein Scalloped mediates transcriptional output of the Hippo growth-regulatory pathway. Dev Cell 14, 388-398.

Xu, T., Wang, W., Zhang, S., Stewart, R.A., and Yu, W. (1995). Identifying tumor suppressors in genetic mosaics: the Drosophila lats gene encodes a putative protein kinase. Development 121, 1053-1063.

Yagi, R., Kohn, M.J., Karavanova, I., Kaneko, K.J., Vullhorst, D., DePamphilis, M.L., and Buonanno, A. (2007). Transcription factor TEAD4 specifies the trophectoderm lineage at the beginning of mammalian development. Development 134, 3827-3836.

Yu, J., Zheng, Y., Dong, J., Klusza, S., Deng, W.M., and Pan, D. (2010). Kibra functions as a tumor suppressor protein that regulates Hippo signaling in conjunction with Merlin and Expanded. Dev Cell 18, 288-299.

Yue, T., Tian, A., and Jiang, J. (2012). The cell adhesion molecule echinoid functions as a tumor suppressor and upstream regulator of the hippo signaling pathway. Dev Cell 22, 255-267.

Zender, L., Spector, M.S., Xue, W., Flemming, P., Cordon-Cardo, C., Silke, J., Fan, S.T., Luk, J.M., Wigler, M., Hannon, G.J., et al. (2006). Identification and validation of oncogenes in liver cancer using an integrative oncogenomic approach. Cell 125, 1253-1267.

Zhang, H., Liu, C.Y., Zha, Z.Y., Zhao, B., Yao, J., Zhao, S., Xiong, Y., Lei, Q.Y., and Guan, K.L. (2009a). TEAD transcription factors mediate the function of TAZ in cell growth and epithe- 
lial-mesenchymal transition. J Biol Chem 284, 13355-13362.

Zhang, H., Pasolli, H.A., and Fuchs, E. (2011). Yes-associated protein (YAP) transcriptional coactivator functions in balancing growth and differentiation in skin. Proc Natl Acad Sci U S A 108, 2270-2275.

Zhang, J., Ji, J.Y., Yu, M., Overholtzer, M., Smolen, G.A., Wang, R., Brugge, J.S., Dyson, N.J., and Haber, D.A. (2009b). YAP-dependent induction of amphiregulin identifies a non-cell-autonomous component of the Hippo pathway. Nat Cell Biol 11, 1444-1450.

Zhang, L., Ren, F., Zhang, Q., Chen, Y., Wang, B., and Jiang, J. (2008). The TEAD/TEF family of transcription factor Scalloped mediates Hippo signaling in organ size control. Dev Cell 14, 377-387.

Zhang, N., Bai, H., David, K.K., Dong, J., Zheng, Y., Cai, J., Giovannini, M., Liu, P., Anders, R.A., and Pan, D. (2010). The Merlin/NF2 tumor suppressor functions through the YAP oncoprotein to regulate tissue homeostasis in mammals. Dev Cell 19, 27-38.

Zhao, B., Li, L., Lu, Q., Wang, L.H., Liu, C.Y., Lei, Q., and Guan, K.L. (2011). Angiomotin is a novel Hippo pathway component that inhibits YAP oncoprotein. Genes Dev 25, 51-63.

Zhao, B., Li, L., Tumaneng, K., Wang, C.Y., and Guan, K.L. (2010). A coordinated phosphorylation by Lats and CK1 regulates YAP stability through SCF(beta-TRCP). Genes Dev 24, 72-85.

Zhao, B., Li, L., Wang, L., Wang, C.Y., Yu, J., and Guan, K.L. (2012). Cell detachment activates the Hippo pathway via cytoskeleton reorganization to induce anoikis. Genes Dev 26, 54-68.
Zhao, B., Wei, X., Li, W., Udan, R.S., Yang, Q., Kim, J., Xie, J., Ikenoue, T., Yu, J., Li, L., et al. (2007). Inactivation of YAP oncoprotein by the Hippo pathway is involved in cell contact inhibition and tissue growth control. Genes Dev 21, 2747-2761.

Zhao, B., Ye, X., Yu, J., Li, L., Li, W., Li, S., Yu, J., Lin, J.D., Wang, C.Y., Chinnaiyan, A.M., et al. (2008). TEAD mediates YAP-dependent gene induction and growth control. Genes Dev 22, 1962-1971.

Zhou, D., Conrad, C., Xia, F., Park, J.S., Payer, B., Yin, Y., Lauwers, G.Y., Thasler, W., Lee, J.T., Avruch, J., et al. (2009). Mst1 and Mst2 maintain hepatocyte quiescence and suppress hepatocellular carcinoma development through inactivation of the Yap1 oncogene. Cancer Cell 16, 425-438.

Zhou, D., Zhang, Y., Wu, H., Barry, E., Yin, Y., Lawrence, E., Dawson, D., Willis, J.E., Markowitz, S.D., Camargo, F.D., et al. (2011a). Mst1 and Mst2 protein kinases restrain intestinal stem cell proliferation and colonic tumorigenesis by inhibition of Yes-associated protein (Yap) overabundance. Proc Natl Acad Sci U S A 108, E1312-E1320.

Zhou, Z., Hao, Y., Liu, N., Raptis, L., Tsao, M.S., and Yang, X. (2011b). TAZ is a novel oncogene in non-small cell lung cancer. Oncogene 30, 2181-2186.

Ziosi, M., Baena-López, L.A., Grifoni, D., Froldi, F., Pession, A., Garoia, F., Trotta, V., Bellosta, P., Cavicchi, S., and Pession, A. (2010). dMyc functions downstream of Yorkie to promote the supercompetitive behavior of hippo pathway mutant cells. PLoS Genet 6, 6. 